\title{
Reconstrucción de pared esofágica con colgajos musculares
}

\section{Reconstruction of the esophageal wall with muscle flaps}

\author{
Martha Milena Alfonso, MD. (1); Elkin Benítez, MD. (2); Julio César Granada, MD. ${ }^{(3)}$; \\ Francisco EdUARdo ANAYA, MD. ${ }^{(4)}$; GERMÁN JUNCA, MD. ${ }^{(5)}$; JORGE AlberTo CARRILlO, MD. ${ }^{(6)}$
}

\section{Resumen}

La perforación esofágica concomitante con perforación aórtica secundaria a cuerpo extraño, es una patología poco frecuente que condiciona alta mortalidad. Se han descrito muy pocos casos que hayan recibido manejo híbrido y resultados satisfactorios, en especial cuando el diagnóstico es tardío. En este documento se presenta un caso de perforación esofágica torácica y aórtica secundaria a cuerpo extraño diagnosticado en forma tardía, que recibió manejo híbrido con reparación aórtica vía endovascular, stent esofágico autoexpandible y reconstrucción de la pared esofágica con colgajo muscular sin reparación primaria.

Palabras clave: perforación esofágica, espina de pescado, cuerpo extraño, perforación aórtica, stent, colgajo quirúrgico.

\begin{abstract}
Esophageal perforation accompanying aortic perforation by foreign body is an uncommon condition whose mortality is high. Very few cases that have received hybrid management with satisfactory results have been described, especially when diagnosis is delayed. In this article we present a case of perforation of the thoracic esophagus and aorta by a foreign body, whose diagnosis was delayed. Hybrid management by endovascular aortic repair, self-expandable esophageal stent, and reconstruction of the esophageal wall with a muscle flap, without primary repair.
\end{abstract}

Keywords: esophageal perforation, fish bone, foreign body, aortic perforation, stent, surgical flap.

\section{Introducción}

En 1723, Boerhaave reportó el primer caso de perforación esofágica y desde entonces sigue siendo una patología que condiciona alta mortalidad (1).

Su evaluación y manejo son un tema controversial. Adicionalmente, la perforación esofágica secundaria a cuerpo extraño es una patología poco frecuente; usualmente es mortal, en especial cuando se asocia además a perforación aórtica (2). Puede poner en riesgo la vida del paciente, de ahí que el diagnóstico deba ser oportuno y adecuado. Cuando la perforación esofágica se asocia a fístula aorto-esofágica, se manifiesta, en general, con dolor torácico, disfagia y hematemesis. Puede haber un intervalo asintomático, pero
Hospital Universitario Mayor (HUM) Méderi. Servicio Cirugía de Tórax, Cuidado Intensivo, Gastroenterología y Radiología torácica. Bogotá, Colombia.

(1)Residente de Cirugía General, Universidad del Rosario. Bogotá, Colombia.

${ }^{(2)}$ Residente de Cirugía General, Universidad del Rosario. Bogotá, Colombia.

${ }^{(3)}$ Cirujano de Tórax y de Trasplante pulmonar Hospital Universitario Mayor Méderi. Universidad del Rosario. Bogotá, Colombia. Fundación Santa Fe de Bogotá. Universidad de los Andes.

${ }^{(4)}$ Médico Intensivista, Hospital Universitario Mayor Méderi. Universidad del Rosario. Bogotá, Colombia.

${ }^{(5)}$ Médico Gastroenterólogo, Hospital Universitario Mayor Méderi. Universidad del Rosario. Bogotá, Colombia.

${ }^{(6)}$ Médico radiólogo. Hospital Universitario Mayor Méderi. Universidad del Rosario. Bogotá, Colombia

Correspondencia: Martha Milena Alfonso, correo electrónico: marthamil2@hotmail.com Recibido: 13/09/16. Aceptado: 10/11/16. 
luego suele presentarse hemorragia de vías digestivas altas masiva y catastrófica que lleva a exanguinación en las siguientes horas o días. Pocos pacientes sobreviven $(2,4)$.

El caso expuesto es de valioso interés científico ya que se propone un manejo menos agresivo en el que se aprovechan los avances tecnológicos que existen en la actualidad incluyendo los abordajes endoscópicos, toracoscópicos y percutáneos, que podrían mejorar la sobrevida en esta patología.

\section{Caso}

Paciente de sexo femenino, de 54 años, quien ingresó al servicio de urgencias remitida de institución de II nivel de complejidad por cuadro clínico de dolor torácico y epigástrico de 9 días de evolución, irradiado al dorso, de intensidad 10/10. Al revisar la historia clínica extrainstitucional, se encontró que inicialmente recibió manejo médico para dispepsia y se descartó síndrome coronario agudo y patología esofágica a través de endoscopia de vías digestivas (EVDA), que estuvo dentro de los límites normales. Por persistencia del dolor, solicitaron radiografía de tórax en la que se evidenció ensanchamiento mediastinal, que sumado al cuadro clínico sugirió patología aórtica, motivo por el cual realizaron angioTAC. Ingresó a nuestra institución taquicárdica, hemodinámicamente estable, con examen físico normal. Como antecedentes de importancia refirió diabetes mellitus tipo 2 en tratamiento con medicina alternativa, dos cesáreas y alergia a la penicilina, y negó historia de sangrado digestivo alto o bajo. La angio-TAC sugirió ruptura contenida de la pared lateral derecha de la aorta torácica a nivel de la carina, con engrosamiento de las paredes del esófago proximal y derrames pleurales bilaterales libres en escasa cantidad (figura 1a). Fue llevada a corrección endovascular de ruptura aórtica torácica, con endoprótesis recubierta (MEDTRONIC VALIANT VAMF2222C 150TE), la cual se desplegó respetando el ostium de la arteria subclavia izquierda con cubrimiento exitoso del sitio de ruptura, sin evidencia de extravasación posterior del medio de contraste (figura 1b).

En el segundo día postoperatorio presentó taquicardia y disnea sin causa aparente, hecho que motivó la exposición del caso en junta quirúrgica y médica interdisciplinaria. Se interrogó nuevamente a la paciente quien refirió ingesta de pescado 12 días antes de los síntomas y posterior sensación de cuerpo extraño. Se revisaron nuevamente las imágenes de ingreso, que como ya se mencionó incluían una EVDA normal.

Se decidió efectuar una nueva angio-TAC torácica con doble contraste en la que se observó stent
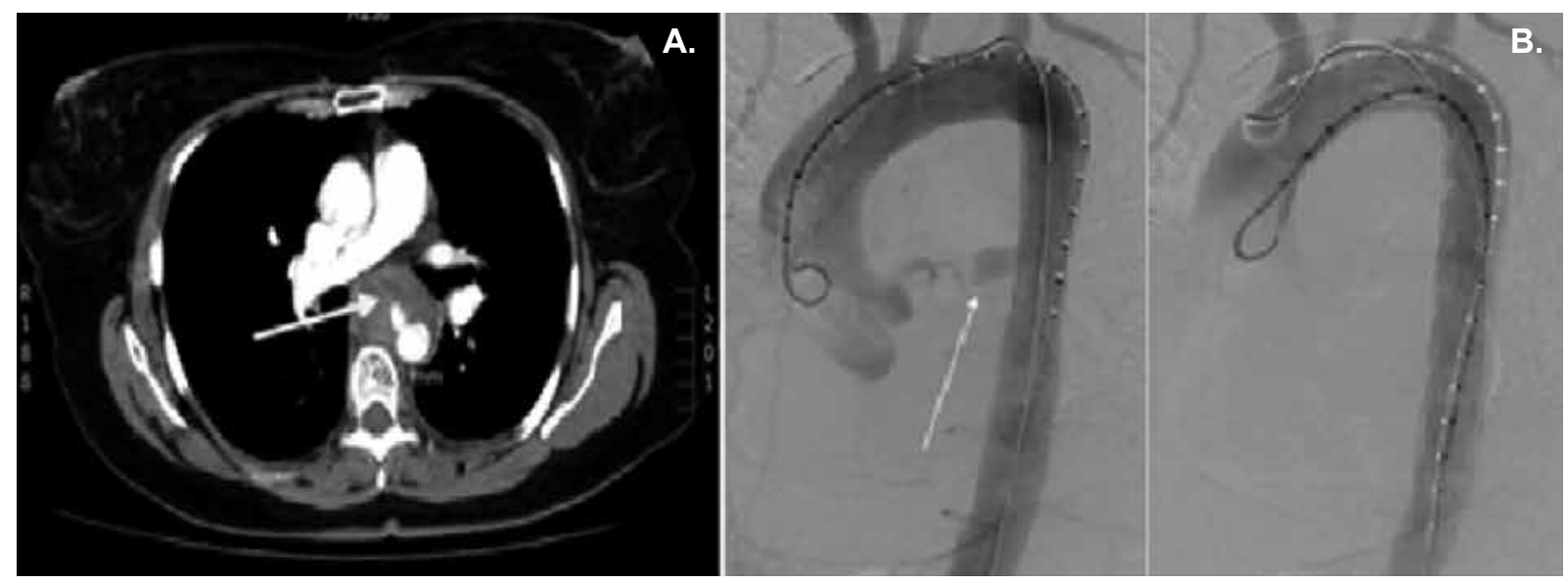

Figura 1. (A). Angiotomografía torácica con imagen de falso aneurisma y gran hematoma en aorta torácica descendente. (B). Corrección endovascular de ruptura aórtica torácica con endoprótesis desplegándose sobre el ostium de la subclavia izquierda. 
en aorta descendente, extravasación del medio de contaste oral por la pared posterior del esófago, neumomediastino y derrames pleurales bilaterales que confirmaron una perforación esofágica (figura 2).

Ante estos hallazgos se inició manejo antibiótico de amplio espectro y se hizo nueva endoscopia de vías digestivas altas. Se encontró una solución de continuidad en la pared posterior del esófago medio, con foco de necrosis transmural de $2,5 \mathrm{~cm}^{2}$ a $29 \mathrm{~cm}$ de la arcada dental; se implantó un stent recubierto de $120 \mathrm{~mm} \times 18 \mathrm{~mm}$ en posición adecuada, con borde proximal a $25 \mathrm{~cm}$ de la arcada dentaria (figura 3 ).

Inmediatamente después del implante del stent esofágico, se llevó a cirugía para drenaje de abundante líquido seropurulento mediastinal por videotoracoscopia. Se halló ruptura esofágica posterior de $5 \mathrm{~cm}$ que comprometía el 50\% de la circunferencia, con bordes necróticos y pérdida de tejido. El stent autoexpandible se encontraba desplazado dejando libre una longitud de $2 \mathrm{~cm}$, así que se decidió realizar toracotomía anterolateral derecha para desbridamiento de bordes y reconstrucción de la pared esofágica con dos colgajos musculares pediculados intercostales (séptimo y octavo). Fue imposible realizar reparación primaria por el grado de infección,

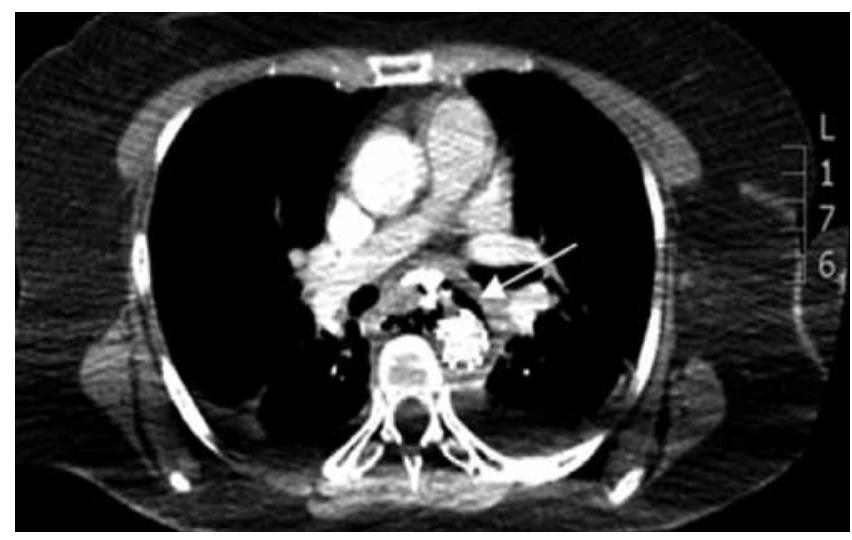

Figura 2. Angiotomografía de tórax con doble contraste. Flecha que indica extravasación del medio de contraste de la pared posterior del esófago y neumomediastino.

necrosis de los bordes y pérdida de la pared posterior (figura 4). Se dejaron dos tubos de tórax derechos, uno anterior, otro posterior y uno izquierdo.

Inicialmente se trató sin vía oral, nutrición parenteral, antibioticoterapia de amplio espectro, antimicótico y vigilancia integral en cuidado intensivo.

En la evolución postoperatoria se generó una fístula esófago-cutánea organizada hacia la toracostomía derecha documentada en un esofagograma de control.

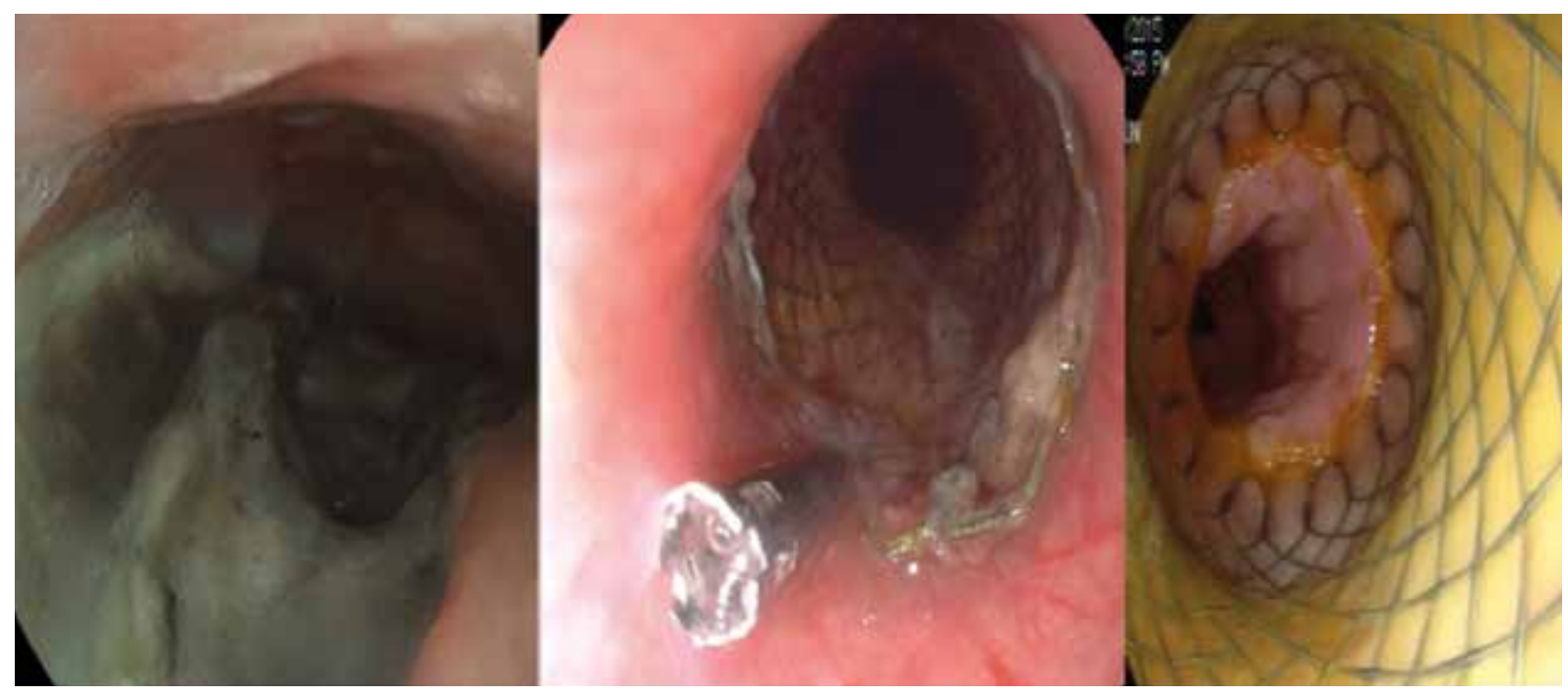

Figura 3. Endoscopia de vías digestivas. Solución de continuidad en pared posterior del esófago medio con foco de necrosis transmural de $2,5 \mathrm{~cm}^{2}$, con implante de stent esofágico cubierto. 
Posteriormente, se hizo cambio a nutrición enteral con yeyunostomía, y se reacomodó el stent esofágico en el postoperatorio. La estancia hospitalaria fue de 83 días dado que requirió cambio en el manejo antibiótico por una sobreinfección con Acinetobacter.

Un mes después de su egreso, se hizo cambio del stent esofágico a uno plástico totalmente recubierto POLYFLEX con el cual permaneció por dos meses, toleró la dieta corriente, y en endoscopia de control se observó mucosa esofágica normal (figura 5a). Seis meses después, luego de retirar el stent POLYFLEX, se practicó esofagograma de control que documentó cierre completo de la fístula.

Siete meses luego de su intervención quirúrgica, ingresó nuevamente por dolor en epigastrio e hipocondrio izquierdo, tos, vómito y un episodio de hematemesis. Se realizó TAC de abdomen que reportó infartos esplénicos y renales bilaterales y TAC de tórax que mostró endoprótesis endovascular en el trayecto de la aorta descendente, leve dilatación del esófago y hallazgos sugestivos de cámara de hidroneumotórax tabicada residual. Se consideró embolia séptica secundaria a prótesis aórtica, por lo que fue llevada a retiro de la prótesis endovascular por cirugía vascular. De manera intraoperatoria se evidenció infección de la prótesis endovascular aórtica con aortitis severa y trombos sépticos proximales intraluminales. Durante el retiro de la prótesis presentó perforación aórtica y falleció en el transoperatorio.

\section{Discusión}

En el caso expuesto se documenta perforación del esófago medio y de la aorta descendente, en el que inicialmente se detectó perforación aórtica y se trató con endoprótesis. La perforación aórtica asociada a perforación esofágica secundaria a cuerpo extraño es poco frecuente y se localiza usualmente en la aorta ascendente, descendente o en la subclavia izquierda $(5,6)$. Dada la persistencia de la sintomatología y el antecedente de ingesta de cuerpo extraño o atragantamiento con espina de pescado, era alta la sospecha de perforación esofágica secundaria, pese a endoscopia de vías digestivas inicial normal. Se han descrito casos en los cuales existe antecedente de ingesta de espina de pescado, que refieren perforación esofágica hasta dos semanas después de su ingesta (7), hecho que puede ocurrir por erosión gradual de la pared esofágica a causa de un cuerpo extraño impactado.

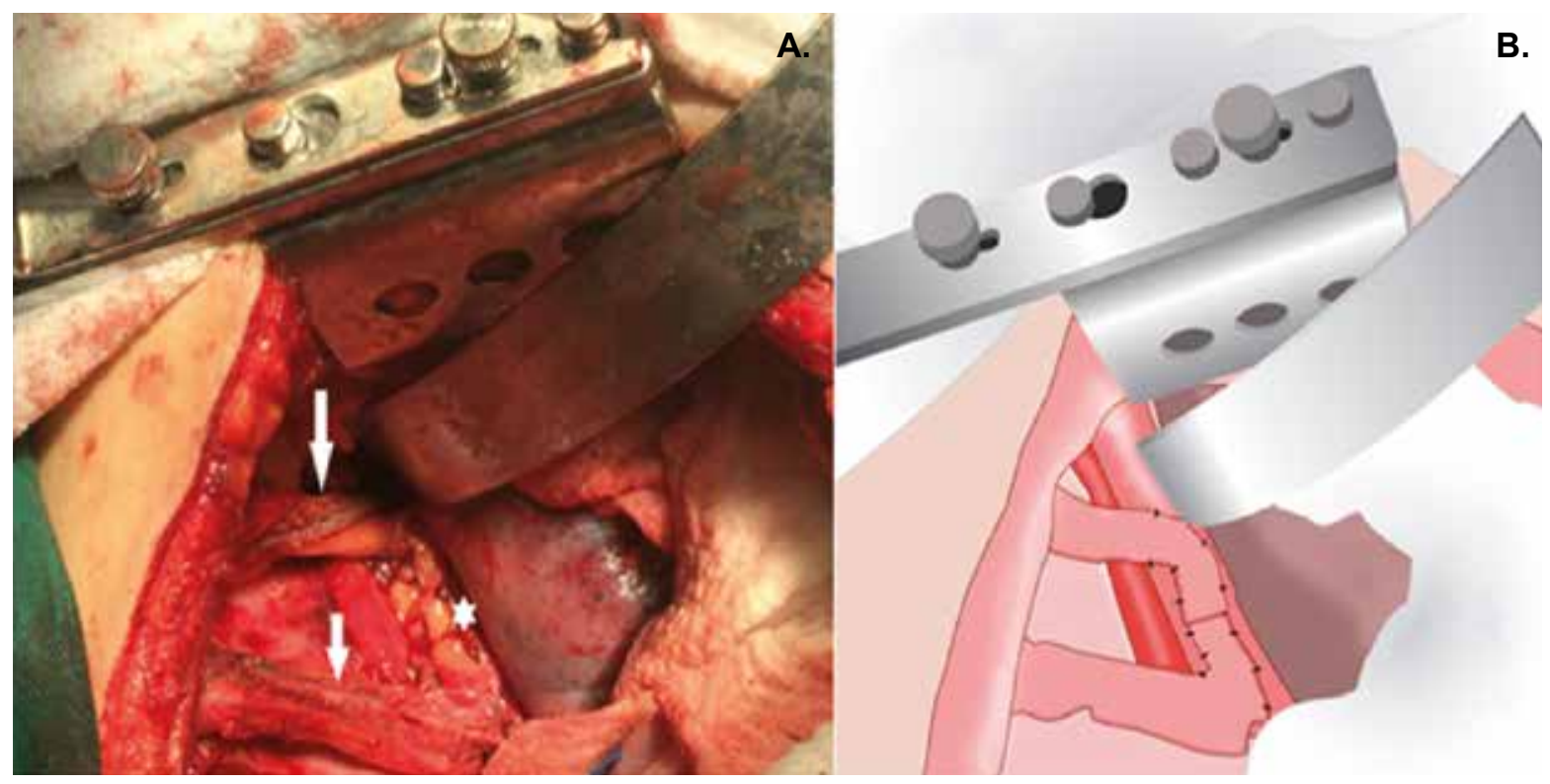

Figura 4. (A) Reconstrucción esofágica con músculo intercostal. Colgajo muscular (flecha), neoesófago (asterisco). (B) Colgajo muscular pediculado de intercostal. 


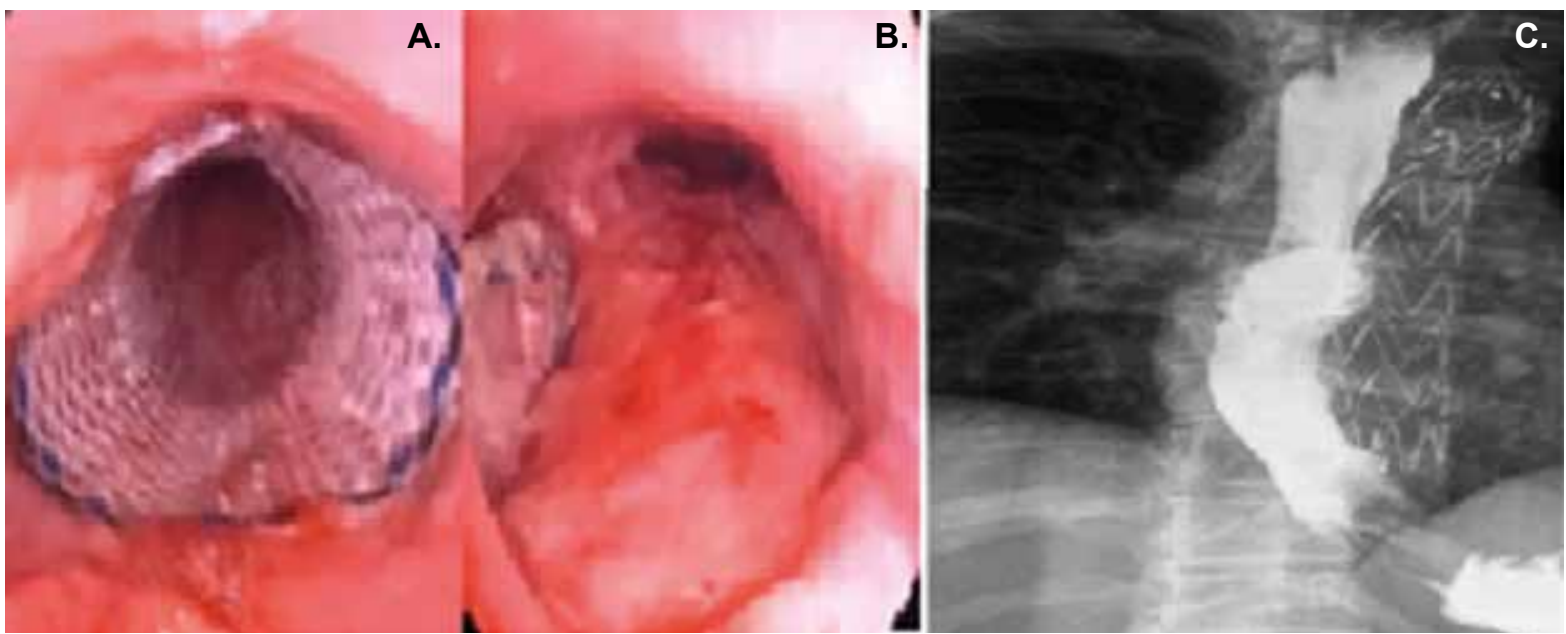

Figura 5. Imágenes de seguimiento. (A) Stent POLYFLEX in situ. (B) Visión endoscópica del colgajo muscular. (C) Esofagograma de control sin evidencia de fugas.

En un estudio de Arantes et al. llevado a cabo en Brasil, durante 7 años, en 143 pacientes con sospecha de lesión esofágica traumática, se realizó endoscopia y se documentó solo un falso negativo que luego fue confirmado en cirugía, describiéndose así sensibilidad del 95,8\%, especificidad del $100 \%$ y valores predictivos positivo y negativo del $100 \%$ y del $99,2 \%$, respectivamente, en la detección de lesión esofágica traumática. Por tanto, una endoscopia normal puede excluir lesión esofágica con elevada precisión (8). La EVDA provee una visualización directa de la lesión y es de gran valor en la evaluación de la perforación esofágica causada por trauma penetrante. Hay reportes de sensibilidad del $100 \%$ y especificidad del $83 \%$ bajo esta condición en otras publicaciones $(1,9)$.

La implantación de los cuerpos extraños en el tracto gastrointestinal usualmente ocurre en la cavidad oral y la laringe, y a este nivel pueden removerse con facilidad; sin embargo, cuando no se detectan y persisten los síntomas, se debe sospechar un cuerpo extraño en el esófago (10). En un estudio hecho por Eliashar et al., en 45 pacientes con alta sospecha, se concluyó que existe una alta tasa de EVDA negativas para la detección de cuerpo extraño esofágico, los cuales sí se observaron en tomografía computarizada multicorte de 64 detectores con una sensibilidad del $96,7 \%$ y especificidad del $100 \%$ (11), mientras se reportó una sensibilidad de tan solo el 51 al 55\% para la radiografía simple (12, 13). En otras series se ha reportado que la tomografía computarizada puede detectar huesos de pescado hasta en el 98,5\% de los casos (10).

El $80-90 \%$ de los cuerpos extraños pasan al estómago (8); usualmente cuando se pueden localizar, están en el esófago cervical. En cuanto a la localización de las perforaciones esofágicas, se ha reportado $50 \%$ de los casos a nivel torácico, $30 \%$ distales y $20 \%$ cervicales (14) y en otros estudios se describe gran porcentaje de localización en el tercio inferior del esófago correspondiente a 56,7$65 \%$, a $21-36,7 \%$ en el medio y a $7,5-14 \%$ en el superior $(15,16)$.

Los mayores avances en el diagnóstico involucran la endoscopia y la tomografía axial computarizada, ya que esta última permite evaluar adicionalmente la severidad de la contaminación mediastinal o pleural.

No se debe olvidar que para el diagnóstico oportuno es importante el alto índice de sospecha en la atención médica primaria, lo cual se logra con la elaboración de una buena historia clínica. 
El manejo híbrido consiste en el uso conjunto y secuencial de técnicas radiológicas o endoscópicas y cirugía abierta o mínimamente invasiva. En la expresión de la evolución en el abordaje de la perforación esofágica, se han expandido significativamente las opciones de manejo en esta patología y ha mejorado la morbilidad y mortalidad (17). Involucra técnicas radiológicas para colocación de drenes en pacientes con abscesos loculados o colecciones no drenadas después de la reparación primaria. El abordaje toracoscópico se puede usar para drenaje e incluso para decorticación pulmonar en caso de ser necesario, en conjunto con métodos endoscópicos como la colocación de clips o stents para cierre de perforación (17).

El caso que se expone es un claro ejemplo de manejo híbrido en una perforación esofágica, pues inicialmente se hizo tratamiento endoscópico con stent esofágico y luego manejo quirúrgico, en un comienzo toracoscópico y luego abierto con reconstrucción esofágica con colgajo muscular y drenaje.

La EVDA puede hacer diagnóstico y tratamiento, así que se considera una herramienta clave para la evaluación de la perforación esofágica. Desempeña un rol importantísimo dado que permite detectar el cuerpo extraño y su localización, evaluar la viabilidad de la mucosa esofágica, la extensión de la lesión y si hay patología secundaria, además de realizar intervencionismo para manejo definitivo, cierre de fugas con clips o suturas vía endoscópica, uso de stent que realice una exclusión de la fuga y/o drenaje endoluminal de la infección mediastinal (18, 19, 20); incluso es útil para facilitar la colocación de sondas para nutrición enteral (21). Con el paso del tiempo, la valoración y el tratamiento endoscópico han aumentado desde un $37 \%$ en 1990 a 1994 hasta un $80 \%$ en 2005 a 2009 (20).

Se recomienda que todos los pacientes se aborden de forma inicial con endoscopia, en esencia por dos razones: identificar patología secundaria y ofrecer posible manejo no quirúrgico (18).

Las perforaciones limitadas o iatrogénicas detectadas en forma temprana son buenas opciones para manejo endoscópico. El tratamiento híbrido se indica en pacientes con perforación extensa y gran cantidad de contaminación torácica o abdominal. Así mismo, se ha descrito el uso exitoso de endoclips en defectos anastomóticos menores del $25 \%$ de la circunferencia o menores de $2 \mathrm{~cm}$ (22).

En 1990 se reportó el uso de stents metálicos parcialmente recubiertos que originalmente se adoptaron solo para el uso en pacientes no candidatos para cirugía. El único inconveniente era su remoción posterior, difícil e incluso imposible dado que sus extremos se pueden incrustar profundamente en la pared esofágica. Por esto, en la actualidad su colocación puede ser segura pero solo de forma temporal, por un número limitado de semanas (23). Cuanto menos tiempo permanezca el stent en el esófago, más fácil será su extracción. Se recomienda, de igual forma, que un stent metálico parcialmente recubierto no permanezca por más de seis a ocho semanas en el esófago para evitar complicaciones secundarias, que pueden involucrar sangrado, fracturas del stent, obstrucción, impactación, fístula aorto-esofágica e incluso abscesos epidurales. Los stents completamente recubiertos, plásticos, tienen menor riesgo de complicaciones durante su extracción $(23,24)$.

Los stents disponibles en el mercado pueden ser total o parcialmente recubiertos, metálicos o plásticos; entre ellos figuran: Ultraflex ${ }^{\circledR}$, Flamingo Wallstent $^{\circledR}$, Choostent TM $^{\circledR}$, Niti-S ${ }^{\circledR}$, FerX-Ella ${ }^{\circledR}$ stent, Hanarostent ${ }^{\circledR}$, Alimaxx ${ }^{\circledR}$, WallFlex ${ }^{\circledR}$ y Polyflex ${ }^{\circledR}$. En manos expertas su implantación se asocia con riesgo bajo de complicaciones y alta tasa de éxito $(1,17,23)$.

Freeman et al. reportaron tratamiento exitoso en una serie de 16 pacientes con ruptura iatrogénica del esófago que llevaban de 3 a 121 horas de evolución, usando un stent plástico autoexpandible (polyflex), con un rango de migración del $18 \%$, facilidad de recuperación de 17 a 91 días, estancia hospitalaria de 3 a 38 días y cero mortalidad; el stent fue retirado a los 17 a 91 días (25). Existe otro reporte en donde 11 pacientes con perforación del esófago distal fueron tratados con stents metálicos parcialmente recubiertos (Ultraflex o FlamingoWallstent), con un intervalo desde la perforación hasta la colocación de stent entre 24 horas hasta 28 días, de los cuales solo 2 requirieron esofagectomía por múltiples abscesos, 
7 permanecieron con los stents por 7 semanas y 2 tuvieron los stent Flamingo por 14 semanas con una extracción más difícil de realizar dado que se encontraban profundamente incrustados en la pared esofágica; solo documentaron 1 caso de migración hacia el estómago (24). En el caso que se expone, el stent permaneció desplazado por varios días, y su reacomodación no se llevó a cabo inmediatamente dado que se corría el riesgo de dañar los colgajos.

Van Hell et al., reportaron en una serie de casos de un total de 33 pacientes, 19 de ellos con perforación, que fueron tratados con éxito con stent metálico autoexpandible, y reportaron un $97 \%$ de recuperación de la misma (23). Se recomienda que la parte cubierta del stent se extienda al menos $3 \mathrm{~cm}$ o más sobre el margen proximal de la perforación (1).

Hay múltiples series de casos de colocación de stents esofágicos en perforación y fuga anastomótica esofágica con buenos resultados (8). No hay evidencia que el tratamiento o diagnóstico endoscópico en pacientes con perforación esofágica aumente la contaminación pleural o mediastinal (21), ni se encontraron reportes que mencionen la implantación del stent como factor agravante de una perforación secundaria a cuerpo extraño. Sin embargo, en el caso que aquí se publica, la paciente tenía una perforación de tan solo $2,5 \mathrm{~cm}$ y luego de la colocación del stent se evidenció, de manera intraoperatoria, una ruptura de $5 \mathrm{~cm}$, de lo cual se puede deducir que las paredes esofágicas donde se encontraba la lesión estaban friables con probable disminución de la perfusión de sus bordes a pesar de su estabilidad hemodinámica, de modo que al momento de expandir el stent se pudo generar una perforación mayor.

Los principios de manejo de la perforación esofágica han cambiado dramáticamente hacia un manejo con tendencia más conservadora que incluye el uso de stents en la mayoría de casos.

No hay consenso en la literatura acerca del manejo quirúrgico determinado como estándar de oro en la perforación esofágica, pues este varía significativamente con base en factores como la presencia de patología secundaria, necrosis de la pared, sepsis, estabilidad del paciente, tiempo de evolución, localización de la perforación y tamaño de la lesión $(17,21,26)$. La decisión de elección entre las diferentes opciones quirúrgicas se debe individualizar según cada caso.

Dentro de las diferentes opciones quirúrgicas están el drenaje, la decorticación, la reparación primaria con o sin refuerzo con colgajos, la reparación con tubo en t para el control de la fístula, la resección esofágica o la exclusión esofágica con esofagostomía cervical, el grapado del esófago distal y la gastrostomía o preferiblemente la yeyunostomía para nutrición enteral (17).

De otra parte, cuando hay contaminación pleural es indispensable el drenaje, que usualmente se implementa en pacientes con perforación tardía y puede llegar a hacerse mediante radiología intervencionista (17). La decorticación consiste en retirar la pleura visceral y se lleva a cabo en pacientes con colapso pulmonar extenso y contaminación intrapleural.

La reparación primaria consiste en cerrar la lesión esofágica. Se realiza en todos los casos en los que el tamaño de la perforación y la viabilidad del tejido lo permitan, e independiente del intervalo entre la perforación y la cirugía (27). Antiguamente, se identificaban las primeras 24 horas como el intervalo aceptable para la reparación primaria; sin embargo, en la literatura varias revisiones reportan alta frecuencia en el diagnóstico tardío y documentan mejoría en la supervivencia con reparación primaria, incluso para lesiones reconocidas luego de las primeras 24 horas $(16,27)$. Adicionalmente, se ha descrito que la valoración endoscópica de la viabilidad de la mucosa esofágica es más importante que el tiempo de evolución (18). La mejoría de los resultados, entre ellos la disminución de los niveles históricos de morbilidad, mortalidad y costos, depende de que el paciente reciba atención apropiada a cargo de un equipo quirúrgico experimentado (18).

Dentro del manejo quirúrgico en la perforación esofágica siempre se incluye el desbridamiento de tejido necrótico y edematoso antes de intentar la reparación primaria, especialmente en los casos que llevan más de 24 horas (28). 
En un estudio de la Universidad de Michigan se efectuó reparación primaria a todas las perforaciones esofágicas no malignas cuando el esófago era rescatable independiente del intervalo entre la lesión y la intervención. De un total de 37 pacientes con perforación esofágica, 22 recibieron reparación primaria, se expuso la lesión y se hizo desbridamiento del tejido necrótico y sutura en dos capas, mucosa y submucosa, con sutura absorbible 4-0 y aproximación de la capa muscular con sutura 3-0 continua o separada. El 95\% de los pacientes a quienes se les realizó reparación primaria independiente del tiempo de evolución, sobrevivieron (27).

Por otro lado, en algunas instituciones se considera dentro de las guías de manejo la esofagectomía en casos de sepsis cuando no es posible la reparación primaria (15). En algunos artículos describen que la experiencia ha demostrado que la sutura directa de la mucosa y la capa muscular por sí sola, incluso dentro de las primeras 24 horas de la ruptura, no es tan confiable, pues puede no resistir las fluctuaciones en los cambios de presión intraesofágica o no contener líquidos dados por vía oral días después de la cirugía, y probablemente los tejidos edematosos resulten friables luego de la exposición prolongada al ácido gástrico y a los jugos digestivos; esto, en conjunto con una condición crítica del paciente, después de un tiempo tardío de diagnóstico, hace que muchos cirujanos opten por la esofagectomía y el lavado de la cavidad pleural, en lugar de la cirugía reconstructiva (28). No obstante, se ha demostrado que la reparación primaria es aplicable en perforaciones intratorácicas no malignas, independiente del intervalo entre la lesión y la cirugía dado que disminuye la mortalidad, acorta la estancia hospitalaria y tiene buenos resultados funcionales $(14,26,27)$.

Sin embargo, el caso expuesto es único dado que a pesar de la extensión de la lesión y teniendo en cuenta el estado clínico general de la paciente y la ausencia de patología secundaria esofágica, se logró preservar el esófago con una reconstrucción esofágica completa, mediante el uso de colgajos de músculos intercostales apoyados con endoprotesis esofágica a pesar de que no se pudo hacer reparación primaria, lográndose comprobar la incorporación total del colgajo a la pared esofágica con formación adecuada de mucosa en la superficie endoluminal.

El reforzamiento de la reparación primaria se puede lograr con el uso de pleura, grasa pericárdica, colgajo de músculo intercostal o diafragmático, estómago, omento e incluso con malla absorbible y pegamento de fibrina $(16,17,29)$. Se ha descrito el abordaje transtorácico en un 59\% de los casos, seguido del transabdominal con un $37 \%$ y el toracoabdominal con un $4 \%$ (18).

La pleura y el pericardio no son lo suficientemente gruesos ni vascularizados y predisponen a estrechez tardía. Petrovsky fue el primero que describió el uso del diafragma en cirugías del esófago en 1948 (30). El omento, el diafragma y el estómago requieren laparotomía y tienen aplicación limitada al esófago distal. Entre tanto, el uso de colgajo de músculo intercostal fue descrito inicialmente por Penton y Bratingan en 1951 en experimentos animales; sin embargo, en la actualidad está muy poco documentado en la literatura. En 1997 se describieron dos casos de reparación usen los que usó músculo intercostal pediculado (31). El músculo intercostal es vascularizado y se puede aplicar en el tercio medio y superior del esófago, y puede ser llevado al sitio de la lesión y fijado mediante suturas no absorbibles o material de absorción lenta. Es grueso, fuerte y tiene baja probabilidad de degradarse o formar estrecheces. La desventaja es que su movilización deja un defecto en la pared torácica (28). El injerto de músculo intercostal pediculado merece más aplicación en el cierre del esófago, particularmente en perforaciones tardías y fístulas postoperatorias (31).

Es claro que para prevenir la contaminación esofágica se debe iniciar antibioticoterapia temprana y soporte nutricional enteral, el cual se puede asegurar con yeyunostomía (15).

Finalmente, tal como se realizó en el caso, una vez se haya drenado, la perforación esofágica puede tratarse como una fístula esofagocutánea igual que cualquier otra fístula gastrointestinal que sanará de manera espontánea, preservando su permeabilidad distal mientras se haga un manejo apropiado 
de la sepsis y del soporte nutricional. En una serie de Martínez se usó un tratamiento descrito como “agresivo conservador" en donde se pudo llevar la perforación esofágica a una fístula esofagocutánea luego del drenaje de líquido pleural con un tubo; se reportó una sobrevida del 100\% (32). En el caso que se expone se formó una fístula esofagocutánea organizada gracias a que se dejó un tubo de tórax cercano al sitio de la lesión.

La presentación clínica de este caso fue inusual dado el tiempo de evolución, el estado general de la paciente y la magnitud de la perforación; genera valioso interés puesto que se presentan varios factores muy poco comunes que usualmente se asocian con alta mortalidad.

En conclusión, el advenimiento de la tecnología ha facilitado el manejo de algunas patologías graves. El tratamiento hibrido es una clara manifestación de cómo la experiencia y las diversas opciones de manejo mejoran los resultados de los pacientes. El éxito involucra un manejo multidisciplinario completo que incluya cuidado intensivo, gastroenterología y radiología, y que esté influenciado por la experiencia del equipo quirúrgico y la habilidad de los equipos endoscópicos y radiológicos para mantenerse al día. Si bien es cierto que cuando han pasado más de 24 horas antes del diagnóstico y hay necrosis de la pared, las complicaciones y la mortalidad aumentan (26), esta última se ve aún más influenciada cuando el tratamiento tarda en manos de médicos sin experiencia para realizar el diagnóstico, en especial cuando los síntomas están enmascarados (33). Se demostró que con los avances en cirugía, en la actualidad es posible mejorar el tiempo de sobrevida de los pacientes con perforación esofágica secundaria a cuerpo extraño y fístula aorto-esofágica, y que poco a poco se logrará que deje de ser una patología mortal. Es importante destacar que luego de la reconstrucción de la pared posterior del esófago con los colgajos, se pudo verificar la incorporación de los mismos al esófago, incluso se apreció el crecimiento de mucosa esofágica, y que después de transcurridos tres meses fue imposible identificar donde se encontraban los colgajos musculares.

\section{Bibliografía}

1. Repici A, Rando G. Stent for nonmalignant leaks, perforations, and ruptures. Techniques in Gastrointestinal Endoscopy. 2010;12(4):237-45.

2. Macchi V, Porzionato A, Bardini R, Parenti A, De Caro R. Rupture of ascending aorta secondary to esophageal perforation by fish bone. J Forensic Sci. 2008;53(5):1181-4.

3. Sica G, Djapardy V, Westaby S, Maynard N. Diagnosis and management of aortoesophageal fistula caused by a foreign body. Ann Thorac Surg. 2004;77:2217-8.

4. Fang Y, Zhou X, Liu J. Aortoesophageal fistula (AEF): Fatal upper gastrointestinal haemorrhage. Turk J Gastroenterol. 2014;25(Suppl.1):321-3.

5. D'Costa H, Bailey F, McGavigan B, George G, Todd B. Perforation of the oesophagus and aorta after eating fish: an unusual cause of chest pain. Emerg Med J. 2003;20:385-6.

6. Kelly S, Peters P, Ogg M, Li A, Smithers B. Successful management of an aortoesophageal fistula caused by a fishbone - case report and review of literature. J Cardiothorac Surg. 2009;4:21.

7. Katsetos MC, Tagbo AC, Lindberg MP, Rosson RS. Esophageal perforation and mediastinitis from fish bone ingestion. South Med J. 2003;96:516-20.

8. Arantes V, Campolina C, Valerio SH, et al. Flexible esophagoscopy as a diagnostic tool for traumatic esophageal injuries. J Trauma. 2009; 66(6):1677-82.

9. HorwitzB, Krevsky B, Buckman RF, Jr, Fisher RS, Dabezies MA. Endoscopic evaluation of penetrating esophageal injuries. Am J Gastroenterol. 1993;88(8):1249-53.

10. Woo SH, Kim KH. Proposal for methods of diagnosis of fish bone foreign body in the esophagus. Laryngoscope. $2015 ; 125(11) 2472-5$.

11. Swinnen J, Eisendrath P, Rigaux J, Kahegeshe L, Lemmers A, Le Moine O. Self-expandable metal stents for the treatment of benign upper GI leaks and perforations. Gastrointestinal endoscopy. 2011;73(5):80-899.

12. Eliashar R, Dano I, Dangoor E, Braverman I, Sichel JY. Computed tomography diagnosis of esophageal bone impaction: a prospective study. Ann Otol Rhino Laryngol. 1999;108:708-10.

13. Park S, Choi DS, Shin HS, Cho JM, Jeon KN, Bae KS, et al. Fish bone foreign bodies in the pharynx and upper esophagus: evaluation with 64-slice MDCT. Acta Radiol. 2014;55(1):8-13.

14. Lang HM, Bruns DH, Schmitz B, Wuerl P. Esophageal perforation: principles of diagnosis and surgical management. Surg Today. 2006;36:332-40.

15. Lindenmann J, Matzi V, Neuboeck N, Anegg U, Maier A, Smolle J, et al. Management of esophageal perforation in 120 consecutive patients: clinical impact of a structured treatment algorithm. J Gastrointest Surg. 2013;17:1036-43. 
16. Bufkin BL, Miller JI, Jr. Mamsour KA. Esophageal perforation: emphasison management. Ann Thoracic Surg. 1996;61:1447-51.

17. Carrott PW, Low D. Advances in the management of esophageal perforation. Thorac Surg Clin. 2011;21:541-55.

18. Kuppusamy M, Hubka M, Felisky C, Carrott P, Kline E, et al. Evolving management strategies in esophageal perforation: Surgeons Using nonoperative Techniques to Improve Outcomes. J Am Coll Surg. 2011;213(1):164-71.

19. Henderson JB, Sorser SA, Atia AN, Catalano MF. Repair of esophageal perforation using a novel endoscopic suturing system. Gastrointest Endosc. 2014;80(3):535-7.

20. Esquivel RG, Raju GS. Endoscopic closure of acute esophageal perforations. Curr Gastroenterol Rep. 2013;15:321.

21. Kuppusamy M, Felisky C, Kozarek RA, Schembre D, Ross A, Gan I, et al. Impact of endoscopic assessment and treatment on operative and non-operative management of acute oesophageal perforation. Brit J Surg. 2011;98:818-24.

22. Raju GS, Tarcin O. Endoscopic management of anastomotic esophageal leaks. Techniques in Gastrointestinal Endoscopy. 2006;8(2):66-71.

23. Van Heel NC, Haringsma J, Spaander MC, Bruno MJ, Kuipers EJ. Short-term esophageal stenting in the management of benign perforations. Am J Gastroenterol. 2010;105(7):1515-20.

24. Siersema PD, Homs M, Haringsma J, Tilanus HW, Kuipers EJ. Use of large-diameter metallic stents to seal traumatic nonmalignant perforations of the esophagus. Gastrointestinal Endoscopy. 2003;58(3):356-61.
25. Freeman RK, Van Woerkom JM, Ascioti AJ. Esophageal stent placement for the treatment of iatrogenic intrathoracic esophageal perforation. Ann Thorac Surg. 2007;83(6):2003-7.

26. Sabuncuoglu M, Benzin F, Dandin O, Cakir T, Sozen I, et al. Rare cause of oesophagus perforation. Int J Surg Case Rep. $2015 ; 6: 138-40$.

27. Whyte RI, Iannettoni MD, Orringer MB. Intrathoracic esophageal perforation. The merit of primary repair. J Thoracic Cardiovasc Surg. 1995;109 (1):140-6.

28. Westaby S. An improved method for primary repair after spontaneous oesophageal perforation. Br J Surg. 1980;67:801-3.

29. Bardaxoglou E, Manganas D, Meunier B, Landen S, Maddern GJ, Campion JP, et al. New approach to surgical management of early esophageal thoracic perforation: primary suture repair reinforced with absorbable mesh and fibrin glue. World J Surg. 1997;21:618-21.

30. Petrovsky BV. The use of diaphragm grafts for plastic operations in thoracic surgery. J Thorac Cardiovasc Surg. 1961;41:348-55.

31. Alexander PV, Hollands M, O'Rourke, Tait N. Intercostal pedicle flap for thoracic oesophageal perforations. Aust NZ J Surg. 1997; 67:133-5.

32. Martinez L, Rivas S, Hernandez F, Avila LF, Lassaletta L, Murcia J, et al. Aggressive conservative treatment of esophageal perforations in children. J Pediatric Surg. 2003;38(5):685-9.

33. Ryom P, Ravn JB, Penninga L, Schmidt S, Iversen MG, Skov-Olsen $\mathrm{P}$, et al. Aetiology, treatment and mortality after oesophageal perforation in Denmark. Dan Med Bull. 2011;58(5):A4267. 